\title{
A FINITE DIFFERENCE APPROXIMATION FOR A NONLINEAR SIZE-STRUCTURED PHYTOPLANKTON AGGREGATION MODEL
}

$\mathrm{BY}$

\author{
AZMY S. ACKLEH AND ROBERT R. FERDINAND
}

Department of Mathematics, University of Southwestern Louisiana, Lafayette, Louisiana 70504-1010

Abstract. We consider a nonlinear model that describes the dynamics of a phytoplankton population with aggregation and competition between individual cells. A finite difference method is developed for approximating the solution of this partial differential equation. The convergence of this approximation to a unique bounded variation solution of the model is proved. Numerical results showing the accuracy of this scheme are presented.

1. Introduction. The growth of an individual cell of phytoplankton is influenced by a variety of factors; two of these are light and nutrients. It is well known (see, e.g., [12]) that in an environment where nutrients are abundant, individual phytoplankton cells divide rapidly resulting in very high population densities. This, in turn, causes a rapid decrease in nutrients due to consumption by phytoplankton and self-shading due to the high density of cells, which results in competition for nutrients and light among these cells.

On the other hand, aggregation of these cells into much larger conglomerates affects the distribution of the phytoplankton total population by increasing the mean volume of a particle, hence removing these aggregates from the surface layer of the ocean at a faster rate due to higher settling velocities (see, $[4,5,8,9,10,11,13,15])$. In this paper, we consider the following nonlinear, nonlocal, initial-boundary value problem that describes the dynamics of a phytoplankton population with aggregation and competition between individual cells:

$$
\begin{cases}u_{t}+(g(x, P(t)) u)_{x}=F(u)-\frac{w(x)}{Z} u, & (t, x) \in(0, T] \times\left(0, x_{\max }\right], \\ g(0, P(t)) u(t, 0)=C(t)+\int_{0}^{x_{\max }} \beta(x, P(t)) u(t, x) d x, & t \in(0, T], \\ u(0, x)=u^{0}(x), & x \in\left[0, x_{\max }\right]\end{cases}
$$

Received March 10, 1997.

1991 Mathematics Subject Classification. Primary 35L60, 65M06, 65M12, 92D25. 
where

$$
F(u)=\frac{1}{2} \int_{0}^{x} \eta(x-y, y) u(t, x-y) u(t, y) d y-u(t, x) \int_{0}^{x_{\max }} \eta(x, y) u(t, y) d y
$$

Here, $u(t, x)$ is the density of aggregates of size $x$ at time $t, P(t)=\int_{0}^{x_{\max }} u(t, x) d x$ is the total number of aggregates at time $t$. The parameters $g$ and $\beta$ are the growth and reproduction rates of individual aggregates, respectively. We remark that to include the effects of competition for resources among individuals, we allow the parameters $g$ and $\beta$ to depend on the total population $P$. The parameter $w$ represents the settling speed of an aggregate of size $x, Z$ represents the depth of the surface layer, and $C$ is the rate of inflow of zero-size individuals from an external source. The term $\frac{w u}{Z}$ represents the loss of particles due to sinking out from the surface layer. The function $\eta(x, y)$ represents the rate at which an aggregate of size $x$ coalesces with an aggregate of size $y$. The first term of $F(u)$ describes the rate at which collisions between particles result in the formation of particles in the size interval $[x, x+d x]$, while the second represents the rate at which these collisions cause these particles to be lost from the same interval.

The model (1.1), when $g=g(x), \beta=\beta(x)$, and $C=0$, was developed and studied in [2]. Therein, the convergence of an approximation scheme and the well-posedness of this autonomous semilinear initial-boundary value problem were established. Our goal here is to extend the existence and uniqueness results to our nonlinear setting presented in (1.1), and to develop a finite difference approximation to this equation. To the best of our knowledge, numerical approximation methods for nonlinear equations of type (1.1) are not available in the literature.

To pursue this study, we use techniques similar to those used to study the wellposedness of nonlinear conservation laws (see, e.g., [7, 14]). For this, we define a weak solution of our model (1.1) to be a bounded measurable function $u$ satisfying:

$$
\begin{aligned}
\int_{0}^{x_{\max }} & u(t, x) \phi(t, x) d x-\int_{0}^{x_{\max }} u^{0}(x) \phi(0, x) d x \\
= & \int_{0}^{t} \int_{0}^{x_{\max }}\left(u \phi_{s}+g u \phi_{x}-\frac{w}{Z} u \phi\right) d x d s \\
& +\int_{0}^{t} \phi(s, 0)\left[C(s)+\int_{0}^{x_{\max }} \beta(x, P(s)) u(s, x) d x\right] d s+\int_{0}^{t} \int_{0}^{x_{\max }} F(u) \varphi d x d s
\end{aligned}
$$

for $t \in[0, T]$ and every test function $\phi \in C^{1}\left((0, T) \times\left(0, x_{\max }\right)\right)$. To continue, we make the following assumptions on our model parameters. We remark that similar conditions were used in $[1,2,3,6]$.

(H1) $u^{0}(x) \in B V\left(0, x_{\max }\right) \cap L_{x}\left(0, x_{\max }\right)$ and $u^{0}(x) \geq 0$.

(H2) $w(x)$ is a nonnegative continuously differentiable function.

(H3) $\beta(x, P)$ is a nonnegative continuously differentiable function with respect to $x$ and $P$. 
(H4) $g(x, P)$ is a twice continuously differentiable function with respect to $x$, and is a continuously differentiable function with respect to $P, g(x, P)>0, x \in$ $\left[0, x_{\max }\right)$ and $g\left(x_{\max }, P\right)=0$.

(H5) $C(t)$ is a nonnegative continuously differentiable function.

(H6) $\sup _{(x . P) \in\left[0 . x_{\max }\right) \times[0, \infty)}\left\{\beta(x, P)-\frac{w(x)}{Z}\right\} \leq \theta_{1}$.

(H7) For any sufficiently small $\delta>0$

$$
\sup _{(x . P) \in\left[0 . x_{\max }\right) \times[0, \infty)}\left|\frac{g(x+\delta, P)-g(x, P)}{\delta}+\frac{w(x)}{Z}\right| \leq \theta_{2} .
$$

(H8) $\eta \in C^{1}\left(\left[0, x_{\max }\right] \times\left[0, x_{\max }\right]\right), \eta(x, y)=\eta(y, x)$ and

$$
\begin{cases}\eta(x, y) \geq 0, & x+y \leq x_{\max } \\ \eta(x, y)=0, & x+y>x_{\max }\end{cases}
$$

Our paper is organized as follows. In Sec. 2 we develop an implicit finite difference scheme to approximate the solution of Eq. (1.1) and establish stability and convergence results. In Sec. 3 the uniqueness of $B V$ weak solutions is discussed. Finally, numerical results are presented in Sec. 4.

2. Convergence of the difference scheme. In this section, we follow similar techniques to those used in $[6,14]$ to obtain subsequential convergence of the finite difference method to a solution of the partial differential equation (1.1). Let $\Delta x=\frac{x_{\max }}{N}$ and $\Delta t=\frac{T}{M}$ describe the space and time mesh size, respectively. Here, the mesh points are $x_{j}=j \Delta x, j=0, \ldots, N$ and $t_{k}=k \Delta t, k=0, \ldots, M$. We denote by $u_{j}^{k}$ and $P^{k}$ the difference approximation of $u\left(t_{k}, x_{j}\right)$ and $P\left(t_{k}\right)$, respectively. Also, we define

$$
g_{j}^{k}=g\left(x_{j}, P^{k}\right), \quad \beta_{j}^{k}=\beta\left(x_{j}, P^{k}\right), \quad w_{j}=w\left(x_{j}\right), \quad C^{k}=C\left(t_{k}\right), \quad \eta_{i, j}=\eta\left(x_{i}, x_{j}\right)
$$

and

$$
h_{j}^{k}=\frac{1}{2} \sum_{m=1}^{j} \eta_{j-m, m} u_{j-m}^{k} u_{m}^{k} \Delta x .
$$

We define the difference operator

$$
D_{h}^{-}\left(u_{j}^{k}\right)=\frac{u_{j}^{k}-u_{j-1}^{k}}{\Delta x}, \quad 1 \leq j \leq N,
$$

and the $l^{1}, l^{\infty}$ norm by

$$
\left\|u^{k}\right\|_{1}=\sum_{j=1}^{N}\left|u_{j}^{k}\right| \Delta x \quad \text { and } \quad\left\|u^{k}\right\|_{\infty}=\max _{j=0,1,2 \ldots . N}\left|u_{j}^{k}\right|
$$


respectively. We approximate Eq. (1.1) using the following finite difference method:

$$
\left\{\begin{array}{l}
\frac{u_{j}^{k+1}-u_{j}^{k}}{\Delta t}+\frac{g_{j}^{k} u_{j}^{k+1}-g_{j-1}^{k} u_{j-1}^{k+1}}{\Delta x}+\frac{w_{j}}{Z} u_{j}^{k+1} \\
\quad+u_{j}^{k+1} \sum_{l=1}^{N} \eta_{j . l} u_{l}^{k} \Delta x=h_{j}^{k}, \quad 1 \leq j \leq N \\
g_{0}^{k} u_{0}^{k+1}=C^{k}+\sum_{i=1}^{N} \beta_{i}^{k} u_{i}^{k+1} \Delta x \\
P^{k+1}=\sum_{j=1}^{N} u_{j}^{k+1} \Delta x
\end{array}\right.
$$

with initial condition

$$
u_{j}^{0}=\frac{1}{\Delta x} \int_{(j-1) \Delta x}^{j \Delta x} u^{0}(x) d x, \quad j=1, \ldots, N
$$

Letting

$$
d_{j}^{k}=1+\frac{\Delta t}{\Delta x} g_{j}^{k}+\Delta t \frac{w_{j}}{Z}+\Delta t \sum_{l=1}^{N} \eta_{j . l} u_{l}^{k} \Delta x
$$

we can write (2.1) as a system of linear equations as follows:

$$
A^{k} \overrightarrow{u^{k+1}}=\overrightarrow{f^{k}}
$$

where

$$
\overrightarrow{u^{k+1}}=\left[u_{0}^{k+1}, u_{1}^{k+1}, \ldots, u_{N}^{k+1}\right], \quad \overrightarrow{f^{k}}=\left[C^{k}, u_{1}^{k}+\Delta t h_{1}^{k}, \ldots, u_{N}^{k}+\Delta t h_{N}^{k}\right]
$$

and

$$
A^{k}=\left[\begin{array}{llllll}
g_{0}^{k} & -\Delta x \beta_{1}^{k} & -\Delta x \beta_{2}^{k} & \ldots & -\Delta x \beta_{N-1}^{k} & -\Delta x \beta_{N}^{k} \\
-\frac{\Delta t}{\Delta x} g_{0}^{k} & d_{1}^{k} & 0 & \ldots & \ldots & 0 \\
0 & -\frac{\Delta t}{\Delta x} g_{1}^{k} & d_{2}^{k} & 0 & \ldots & 0 \\
0 & 0 & -\frac{\Delta t}{\Delta x} g_{2}^{k} & d_{3}^{k} & \ldots & 0 \\
0 & \ldots & \ldots & \ldots & \cdots & 0 \\
0 & \ldots & \ldots & 0 & -\frac{\Delta t}{\Delta x} g_{N-1}^{k} & d_{N}^{k}
\end{array}\right]
$$

Our next task is to show that the system of linear equations given in (2.2) has a unique solution that preserves nonnegativity.

Lemma 2.1. Assume $\Delta x$ and $\Delta t$ are chosen so that

$$
\Delta x \frac{\beta_{j}^{k}}{g_{0}^{k}}+\frac{\Delta t}{\Delta x} g_{j}^{k}\left(1+\frac{\Delta t}{\Delta x} g_{j+1}^{k}+\Delta t \frac{w_{j+1}}{Z}\right)^{-1}<1 \text { for } j=0, \ldots, N-1
$$


and for $j=N, \Delta x \frac{\beta_{N}^{k}}{g_{0}^{k}}<1$. Then Eq. (2.2) has a unique solution and, if $u_{j}^{k} \geq 0$ for $j=1, \ldots, N$ and $C^{k} \geq 0$, then $u_{j}^{k+1} \geq 0$ for $j=1, \ldots, N$.

Proof. The proof follows immediately from using techniques similar to those used in Lemma 2.1 of [6], together with Theorem 7.2, p. 43 in [16].

In our next lemma we establish a bound on the $l^{1}$ norm of our difference approximation.

LEMMA 2.2. Under the assumptions of Lemma 2.1 and assuming that $\theta_{1} \Delta t<1$, we have the following bound on the $l^{1}$ of our approximation $u_{j}^{k}$ :

$$
\left\|u^{k}\right\|_{1} \leq\left(\frac{1}{1-\theta_{1} \Delta t}\right)^{k}\left\|u^{0}\right\|_{1}+\sum_{j=1}^{k}\left(\frac{1}{1-\theta_{1} \Delta t}\right)^{k+1-j}\left|C^{j}\right| \Delta t .
$$

Proof. Following the proof of Lemma 2.2 in [6], since $u_{j}^{k+1} \geq 0$ and since $\left\{\beta(x, P)-\frac{w(x)}{Z}\right\} \leq \theta_{1}$, we multiply Eq. (2.1) by $\Delta x$ and sum over the indices $j=$ $1, \ldots, N$ to get

$$
\begin{aligned}
& \frac{\left\|u^{k+1}\right\|_{1}-\left\|u^{k}\right\|_{1}}{\Delta t} \leq C^{k}+\sum_{j=1}^{N}\left(\beta_{j}^{k}-\frac{w_{j}}{Z}\right) u_{j}^{k+1} \Delta x \\
& +\sum_{j=1}^{N} \frac{\Delta x}{2} \sum_{l=1}^{j} \eta_{j-l, l} u_{j-l}^{k} u_{l}^{k} \Delta x-\sum_{j=1}^{N} \Delta x u_{j}^{k+1} \sum_{l=1}^{N} \eta_{j, l} u_{l}^{k} \Delta x .
\end{aligned}
$$

Letting $z=j-l$ we obtain

$$
\begin{aligned}
& \frac{\left\|u^{k+1}\right\|_{1}-\left\|u^{k}\right\|_{1}}{\Delta t} \leq C^{k}+\sum_{j=1}^{N}\left(\beta_{j}^{k}-\frac{w_{j}}{Z}\right) u_{j}^{k+1} \Delta x \\
& +\sum_{l=1}^{N} \Delta x u_{l}^{k} \sum_{z=1}^{N-l} \eta_{z, l} u_{z}^{k} \Delta x-\sum_{j=1}^{N} \Delta x u_{j}^{k+1} \sum_{l=1}^{N} \eta_{j, l} u_{l}^{k} \Delta x \\
& \leq C^{k}+\sum_{j=1}^{N}\left(\beta_{j}^{k}-\frac{w_{j}}{Z}\right) u_{j}^{k+1} \Delta x \\
& +\|\eta\|_{\infty} \sum_{j=1}^{N}\left[\left(u_{j}^{k}-u_{j}^{k+1}\right)\left(\sum_{l=1}^{N} u_{l}^{k} \Delta x\right)\right] \Delta x \\
& \leq\left|C^{k}\right|+\theta_{1}\left\|u^{k+1}\right\|_{1}+\|\eta\|_{\infty}\left\|u^{k}\right\|_{1}\left(\left\|u^{k}\right\|_{1}-\left\|u^{k+1}\right\|_{1}\right) .
\end{aligned}
$$

Multiplying by $\Delta t$ and doing elementary algebra we arrive at

$$
\left(\left\|u^{k+1}\right\|_{1}-\left\|u^{k}\right\|_{1}\right)\left(1+\Delta t\|\eta\|_{\infty}\left\|u^{k}\right\|_{1}\right) \leq\left|C^{k}\right| \Delta t+\theta_{1} \Delta t\left\|u^{k+1}\right\|_{1} .
$$

Then, using the fact that $\Delta t\|\eta\|_{\infty}\left\|u^{k}\right\|_{1} \geq 0$ we get

$$
\begin{aligned}
\left\|u^{k+1}\right\|_{1}-\left\|u^{k}\right\|_{1} & \leq \frac{\left|C^{k}\right| \Delta t}{1+\Delta t\|\eta\|_{\infty}\left\|u^{k}\right\|_{1}}+\frac{\theta_{1} \Delta t\left\|u^{k+1}\right\|_{1}}{1+\Delta t\|\eta\|_{\infty}\left\|u^{k}\right\|_{1}} \\
& \leq\left|C^{k}\right| \Delta t+\theta_{1} \Delta t\left\|u^{k+1}\right\|_{1}
\end{aligned}
$$


or equivalently

$$
\left\|u^{k+1}\right\|_{1} \leq \frac{\left\|u^{k}\right\|_{1}}{\left(1-\theta_{1} \Delta t\right)}+\frac{\left|C^{k}\right| \Delta t}{\left(1-\theta_{1} \Delta t\right)}
$$

which establishes the stated bound.

The next result shows that the difference approximations are uniformly bounded.

LEMMA 2.3. Under the assumptions of Lemma 2.2 and $\theta_{2} \Delta t<1$, we have the following bound on the $l^{\infty}$-Norm of $u_{j}^{k}$ :

$$
\left\|u^{k}\right\|_{\infty} \leq \max \left\{\left\|u^{0}\right\|_{\infty}\left(\frac{1+\Delta t B_{1}}{1-\theta_{2} \Delta t}\right)^{k}, \frac{\|C\|_{\infty}+\alpha_{2} P_{\max }}{\alpha_{1}}\right\}
$$

where $g(0, P) \geq \alpha_{1},|\beta(x, P)| \leq \alpha_{2}$ for $x \in\left[0, x_{\max }\right], \quad P \in\left[0, P_{\max }\right]$, and $B_{1}=$ $\max _{k}\left(\frac{1}{2}\|\eta\|_{\infty}\left\|u^{k}\right\|_{1}\right)$.

Proof. Note that if $u_{0}^{k+1}=\max _{i=0.1, \ldots, N}\left(u_{i}^{k+1}\right)$ then from the second equation of (2.1) we get

$$
g_{0}^{k}\left|u_{0}^{k+1}\right| \leq\left|C^{k}\right|+\sup \left|\beta\left(x, P^{k}\right)\right|\left\|u^{k+1}\right\|_{1} .
$$

Hence,

$$
\begin{aligned}
\left|u_{0}^{k+1}\right| & \leq \frac{\left|C^{k}\right|+\sup \left|\beta\left(x, P^{k}\right)\right|\left\|u^{k+1}\right\|_{1}}{g_{0}^{k}} \\
& \leq \frac{\|C\|_{\infty}+\alpha_{2} P_{\max }}{\alpha_{1}} .
\end{aligned}
$$

Else, if for some $1 \leq j \leq N, u_{j}^{k+1}=\max _{i=0,1, \ldots, N}\left(u_{i}^{k+1}\right)$ then using Eq. (2.1), we get

$$
\begin{aligned}
& u_{j}^{k+1}-u_{j}^{k}+\frac{\Delta t}{\Delta x}\left(g_{j}^{k} u_{j}^{k+1}-g_{j-1}^{k} u_{j-1}^{k+1}\right)+\Delta t \frac{w_{j}}{Z} u_{j}^{k+1}+\Delta t u_{j}^{k+1} \sum_{l=1}^{N} \eta_{j, l} u_{l}^{k} \Delta x \\
& =\frac{\Delta t}{2} \sum_{l=1}^{j} \eta_{j-l, l} u_{j-l}^{k} u_{l}^{k} \Delta x .
\end{aligned}
$$

Since the last term on the left-hand side of the above equation is nonnegative, we get

$$
u_{j}^{k+1}\left(1+\frac{\Delta t}{\Delta x} g_{j}^{k}+\Delta t \frac{w_{j}}{Z}\right)-\frac{\Delta t}{\Delta x} g_{j-1}^{k} u_{j-1}^{k+1} \leq u_{j}^{k}+\frac{\Delta t}{2} \sum_{l=1}^{j} \eta_{j-l, l} u_{j-l}^{k} u_{l}^{k} \Delta x
$$

Hence,

$$
u_{j}^{k+1}\left(1+\Delta t \frac{w_{j}}{Z}\right)+\Delta t \frac{g_{j}^{k}-g_{j-1}^{k}}{\Delta x} u_{j}^{k+1} \leq u_{j}^{k}+\frac{\Delta t}{2}\|\eta\|_{\infty}\left\|u^{k}\right\|_{\infty}\left\|u^{k}\right\|_{1} .
$$

After rearranging terms we obtain

$$
\left[1+\Delta t\left(\frac{w_{j}}{Z}+\frac{g_{j}^{k}-g_{j-1}^{k}}{\Delta x}\right)\right] u_{j}^{k+1} \leq u_{j}^{k}+\frac{\Delta t}{2}\|\eta\|_{\infty}\left\|u^{k}\right\|_{\infty}\left\|u^{k}\right\|_{1} .
$$


Using (H7) we arrive at

$$
\left(1-\theta_{2} \Delta t\right) u_{j}^{k+1} \leq u_{j}^{k}+\frac{1}{2} \Delta t\|\eta\|_{\infty}\left\|u^{k}\right\|_{\infty}\left\|u^{k}\right\|_{1} .
$$

Since $u_{j}^{k} \leq \max _{i=0,1, \ldots, N}\left(u_{i}^{k}\right)=\left\|u^{k}\right\|_{\infty}$, we get

$$
u_{j}^{k+1} \leq\left\|u^{k}\right\|_{\infty}\left(\frac{1+\Delta t B_{1}}{1-\theta_{2} \Delta t}\right)
$$

which gives the final estimate:

$$
\left\|u^{k}\right\|_{\infty} \leq \max \left\{\left\|u^{0}\right\|_{\infty}\left(\frac{1+\Delta t B_{1}}{1-\theta_{2} \Delta t}\right)^{k}, \frac{\|C\|_{\infty}+\alpha_{2} P_{\max }}{\alpha_{1}}\right\} .
$$

Hence, the desired result is obtained.

Next, we will establish a bound on the total variation of the difference approximations.

Lemma 2.4. Assume that $\Delta t$ satisfies $\max \left(\theta_{1}, \theta_{2}+2 B_{1}\right) \Delta t<1$. Then there exists a constant $B_{2}=B_{2}\left(\left|u^{0}\right|_{B V},|C|_{C^{1}}\right)$ such that for all $k=1, \ldots, M,\left\|D_{h}^{-}\left(u^{k}\right)\right\|_{1} \leq B_{2}$.

Proof. We set $\lambda_{j}^{k}=D_{h}^{-}\left(u_{j}^{k}\right)$ and apply the difference operator $D_{h}^{-}$to our equation (2.1) to get

$$
\begin{aligned}
& \frac{\lambda_{j}^{k+1}-\lambda_{j}^{k}}{\Delta t}+D_{h}^{-}\left(\frac{g_{j}^{k} u_{j}^{k+1}-g_{j-1}^{k} u_{j-1}^{k+1}}{\Delta x}\right) \\
& +D_{h}^{-}\left(\frac{w_{j}}{Z} u_{j}^{k+1}\right)+D_{h}^{-}\left(u_{j}^{k+1} \sum_{l=1}^{N} \eta_{j, l} u_{l}^{k} \Delta x\right) \\
& =D_{h}^{-}\left(\frac{1}{2} \sum_{l=1}^{j} \eta_{j-l, l} u_{j-l}^{k} u_{l}^{k} \Delta x\right), \quad j=2, \ldots, N .
\end{aligned}
$$

Now, for $j=1$ we have

$$
\begin{aligned}
\frac{\lambda_{1}^{k+1}-\lambda_{1}^{k}}{\Delta t} & =\frac{1}{\Delta t}\left[\frac{u_{1}^{k+1}-u_{0}^{k+1}}{\Delta x}-\frac{u_{1}^{k}-u_{0}^{k}}{\Delta x}\right]=\frac{-1}{\Delta x}\left[\frac{u_{0}^{k+1}-u_{0}^{k}}{\Delta t}-\frac{u_{1}^{k+1}-u_{1}^{k}}{\Delta t}\right] \\
& =\frac{-1}{\Delta x}\left(\frac{u_{0}^{k+1}-u_{0}^{k}}{\Delta t}+\frac{w_{1}}{Z} u_{1}^{k+1}+D_{h}^{-}\left(g_{1}^{k} u_{1}^{k+1}\right)\right) \\
& +\frac{1}{\Delta x}\left(\frac{1}{2} \eta_{0,1} u_{0}^{k} u_{1}^{k} \Delta x-u_{1}^{k+1} \sum_{l=1}^{N} \eta_{1, l} u_{l}^{k} \Delta x\right) .
\end{aligned}
$$

Setting for convenience

$$
\begin{gathered}
w_{0}=0, \quad D_{h}^{-}\left(u_{1}^{k+1} \sum_{l=1}^{N} \eta_{1, l} u_{l}^{k} \Delta x\right)=u_{1}^{k+1} \sum_{l=1}^{N} \eta_{1, l} u_{l}^{k}, \\
D_{h}^{-}\left(g_{0}^{k} u_{0}^{k+1}\right)=-\frac{u_{0}^{k+1}-u_{0}^{k}}{\Delta t} \text { and } D_{h}^{-}\left(\frac{1}{2} \eta_{0,1} u_{0}^{k} u_{1}^{k} \Delta x\right)=\frac{1}{2} \eta_{0,1} u_{0}^{k} u_{1}^{k},
\end{gathered}
$$


we obtain

$$
\begin{aligned}
& \frac{\lambda_{1}^{k+1}-\lambda_{1}^{k}}{\Delta t}+D_{h}^{-}\left(\frac{g_{1}^{k} u_{1}^{k+1}-g_{0}^{k} u_{0}^{k+1}}{\Delta x}\right)+D_{h}^{-}\left(\frac{w_{1}}{Z} u_{1}^{k+1}\right)+D_{h}^{-}\left(u_{1}^{k+1} \sum_{l=1}^{N} \eta_{1, l} u_{l}^{k} \Delta x\right) \\
& =D_{h}^{-}\left(\frac{1}{2} \eta_{0,1} u_{0}^{k} u_{1}^{k} \Delta x\right) .
\end{aligned}
$$

Multiplying (2.3) and (2.4) by $\Delta x \operatorname{sgn}\left(\lambda_{j}^{k+1}\right)$ and summing over the indices $j=1, \ldots, N$, we get

$$
\begin{aligned}
& \sum_{j=1}^{N}\left(\frac{\left|\lambda_{j}^{k+1}\right|-\left|\lambda_{j}^{k}\right|}{\Delta t}\right) \Delta x \\
& \quad+\sum_{j=1}^{N} \operatorname{sgn}\left(\lambda_{j}^{k+1}\right) \Delta x\left[D_{h}^{-}\left(\frac{g_{j}^{k} u_{j}^{k+1}-g_{j-1}^{k} u_{j-1}^{k+1}}{\Delta x}\right)+D_{h}^{-}\left(\frac{w_{j}}{Z} u_{j}^{k+1}\right)\right] \\
& \quad+\sum_{j=1}^{N} \operatorname{sgn}\left(\lambda_{j}^{k+1}\right) \Delta x\left[D_{h}^{-}\left(u_{j}^{k+1} \sum_{l=1}^{N} \eta_{j, l} u_{l}^{k} \Delta x\right)-D_{h}^{-}\left(\frac{1}{2} \sum_{l=1}^{j} \eta_{j-l, l} u_{j-l}^{k} u_{l}^{k} \Delta x\right)\right] \leq 0 .
\end{aligned}
$$

Performing similar calculations as in Lemma 2.4 of [6], we get

$$
\begin{aligned}
& \frac{\left\|\lambda^{k+1}\right\|_{1}-\left\|\lambda^{k}\right\|_{1}}{\Delta t} \leq\left|\frac{u_{0}^{k+1}-u_{0}^{k}}{\Delta t}\right|+\max _{j}\left|D_{h}^{-}\left(g_{j}^{k}\right)+\frac{w_{j}}{Z}\right|\left\|\lambda^{k+1}\right\|_{1} \\
& +\max _{j}\left|D_{h}^{-}\left(D_{h}^{-}\left(g_{j}^{k}\right)+\frac{w_{j}}{Z}\right)\right|\left\|u^{k+1}\right\|_{1}+\left|D_{h}^{-} g_{1}^{k}\right|\left|u_{0}^{k+1}\right| \\
& -\sum_{j=1}^{N} \operatorname{sgn}\left(\lambda_{j}^{k+1}\right) \Delta x\left[D_{h}^{-}\left(u_{j}^{k+1} \sum_{l=1}^{N} \eta_{j, l} u_{l}^{k} \Delta x\right)\right] \\
& +\frac{1}{2} \sum_{j=1}^{N} \operatorname{sgn}\left(\lambda_{j}^{k+1}\right) \Delta x\left[D_{h}^{-}\left(\sum_{l=1}^{j} \eta_{j-l, l} u_{j-l}^{k} u_{l}^{k} \Delta x\right)\right] .
\end{aligned}
$$

Our next task is to obtain a bound for the term $\left|\frac{u_{0}^{k+1}-u_{0}^{k}}{\Delta t}\right|$. To this end, since $g_{0}^{k} u_{0}^{k+1}=$ $C^{k}+\sum_{i=1}^{N} \beta_{i}^{k} u_{i}^{k+1} \Delta x$, then proceeding similarly as in the proof of Lemma 2.4 in [6] and 
using summation by parts, we get

$$
\begin{aligned}
& \left|g_{0}^{k}\left(\frac{u_{0}^{k+1}-u_{0}^{k}}{\Delta t}\right)+\left(\frac{g_{0}^{k}-g_{0}^{k-1}}{\Delta t}\right) u_{0}^{k}-\left(\frac{C^{k}-C^{k-1}}{\Delta t}\right)\right| \\
& =\left|\sum_{j=1}^{N}\left[\beta_{j}^{k}\left(\frac{u_{j}^{k+1}-u_{j}^{k}}{\Delta t}\right)+u_{j}^{k}\left(\frac{\beta_{j}^{k}-\beta_{j}^{k-1}}{\Delta t}\right)\right] \Delta x\right| \\
& =\mid \sum_{j=1}^{N}\left[-\beta_{j}^{k}\left(D_{h}^{-}\left(g_{j}^{k} u_{j}^{k+1}\right)+\frac{w_{j}}{Z} u_{j}^{k+1}+u_{j}^{k+1} \sum_{l=1}^{N} \eta_{j, l} u_{l}^{k} \Delta x\right.\right. \\
& \leq\left(\left\|\beta_{x}\right\|_{\infty}\|g\|_{\infty}+\frac{1}{Z}\|\beta\|_{\infty}\|w\|_{\infty}\right)\left\|u^{k+1}\right\|_{1}+\|\beta\|_{\infty}\|g\|_{\infty}\left\|u^{k+1}\right\|_{\infty} \\
& +\left\|\beta_{P}\right\|_{\infty}\left(\left|C^{k-1}\right|+\theta_{1}\left\|u^{k}\right\|_{1}\right)\left\|u^{k}\right\|_{1}+\|\eta\|_{\infty}\|\beta\|_{\infty}\left\|u^{k}\right\|_{1}\left(\left\|u^{k}\right\|_{1}+\left\|u^{k+1}\right\|_{1}\right) .
\end{aligned}
$$

The bound on $\left|\frac{u_{0}^{k+1}-u_{0}^{k}}{\Delta t}\right|$ can easily be established from the above inequality. We now proceed to establish bounds on the fourth and fifth terms of the right-hand side of (2.6). The fourth term in (2.6) can be bounded as follows:

$$
\begin{aligned}
& \sum_{j=1}^{N}\left[D_{h}^{-}\left(u_{j}^{k+1} \sum_{l=1}^{N} \eta_{j, l} u_{l}^{k} \Delta x\right)\right] \operatorname{sgn}\left(\lambda_{j}^{k+1}\right) \Delta x \\
& =\sum_{j=1}^{N}\left[D_{h}^{-}\left(u_{j}^{k+1}\right) \sum_{l=1}^{N} \eta_{j, l} u_{l}^{k} \Delta x+u_{j-1}^{k+1} D_{h}^{-}\left(\sum_{l=1}^{N} \eta_{j, l} u_{l}^{k} \Delta x\right)\right] \operatorname{sgn}\left(\lambda_{j}^{k+1}\right) \Delta x \\
& \leq\left\|\lambda^{k+1}\right\|_{1}\|\eta\|_{\infty}\left\|u^{k}\right\|_{1}+\sum_{j=1}^{N}\left[u_{j-1}^{k+1} \sum_{l=1}^{N} D_{h}^{-}\left(\eta_{j, l}\right) u_{l}^{k} \Delta x\right] \operatorname{sgn}\left(\lambda_{j}^{k+1}\right) \Delta x \\
& \leq\left\|\lambda^{k+1}\right\|_{1}\|\eta\|_{\infty}\left\|u^{k}\right\|_{1}+\left\|\eta_{x}\right\|_{\infty}\left\|u^{k}\right\|_{1}\left\|u^{k+1}\right\|_{1}+\left\|\eta_{x}\right\|_{\infty}\left\|u^{k}\right\|_{1}\left\|u^{k+1}\right\|_{\infty} .
\end{aligned}
$$


Similarly, the fifth term in (2.6) can be bounded as follows:

$$
\begin{aligned}
& \frac{1}{2} \sum_{j=1}^{N}\left[D_{h}^{-}\left(\sum_{l=1}^{j} \eta_{j-l, l} u_{j-l}^{k} u_{l}^{k} \Delta x\right)\right] \operatorname{sgn}\left(\lambda_{j}^{k+1}\right) \Delta x \\
& =\frac{1}{2} \sum_{j=1}^{N} \frac{1}{\Delta x}\left[\sum_{l=1}^{j} \eta_{j-l, l} u_{j-l}^{k} u_{l}^{k} \Delta x-\sum_{l=1}^{j-1} \eta_{j-1-l, l} u_{j-1-l}^{k} u_{l}^{k} \Delta x\right] \operatorname{sgn}\left(\lambda_{j}^{k+1}\right) \Delta x \\
& =\frac{1}{2} \sum_{j=1}^{N}\left[\eta_{0, j} u_{0}^{k} u_{j}^{k}+\sum_{l=1}^{j-1}\left(\frac{\left.\eta_{j-l, l} u_{j-l}^{k}-\eta_{j-1-l, l} u_{j-1-l}^{k}\right)}{\Delta x}\right) u_{l}^{k} \Delta x\right] \operatorname{sgn}\left(\lambda_{j}^{k+1}\right) \Delta x \\
& \leq \frac{1}{2}\|\eta\|_{\infty}\left\|u^{k}\right\|_{\infty}\left\|u^{k}\right\|_{1}+\frac{1}{2} \sum_{j=1}^{N}\left[\sum_{l=1}^{j-1}\left(\frac{\eta_{j-l, l}-\eta_{j-1-l, l}}{\Delta x}\right) u_{j-l}^{k} u_{l}^{k} \Delta x\right] \operatorname{sgn}\left(\lambda_{j}^{k+1}\right) \Delta x \\
& \quad+\frac{1}{2} \sum_{j=1}^{N}\left[\sum _ { l = 1 } ^ { j - 1 } \eta _ { j - 1 - l , l } u _ { l } ^ { k } \left(\frac{\left.\left.u_{j-l}^{k}-u_{j-1-l}^{k}\right) \Delta x\right] \operatorname{sgn}\left(\lambda_{j}^{k+1}\right) \Delta x}{\leq} \frac{1}{2}\|\eta\|_{\infty}\left\|u^{k}\right\|_{\infty}\left\|u^{k}\right\|_{1}+\frac{1}{2} \sum_{j=1}^{N}\left[\sum_{l=1}^{j-1} D_{h}^{-}\left(\eta_{j-l, l}\right) u_{j-l}^{k} u_{l}^{k} \Delta x\right] \operatorname{sgn}\left(\lambda_{j}^{k+1}\right) \Delta x\right.\right. \\
& \quad+\frac{1}{2} \sum_{j=1}^{N}\left[\sum_{l=1}^{j-1} \eta_{j-1-l, l} u_{l}^{k} \lambda_{j-l}^{k} \Delta x\right] \operatorname{sgn}\left(\lambda_{j}^{k+1}\right) \Delta x \\
& +\frac{1}{2} \sum_{l=1}^{N}\left[\sum_{j=l+1}^{N} \eta_{j-1-l, l} u_{l}^{k} \lambda_{j-l}^{k} \Delta x\right] \Delta x . \\
& \leq
\end{aligned}
$$

Setting $j-l=m$ and using the assumption (H7) we get

$$
\begin{aligned}
& \frac{1}{2} \sum_{j=1}^{N}\left[D_{h}^{-}\left(\sum_{l=1}^{j} \eta_{j-l, l} u_{j-l}^{k} u_{l}^{k} \Delta x\right)\right] \operatorname{sgn}\left(\lambda_{j}^{k+1}\right) \Delta x \\
& \leq \frac{1}{2}\|\eta\|_{\infty}\left\|u^{k}\right\|_{\infty}\left\|u^{k}\right\|_{1}+\frac{1}{2} \sum_{l=1}^{N}\left[\sum_{m=1}^{N} D_{h}^{-}\left(\eta_{m, l}\right) u_{m}^{k} u_{l}^{k} \Delta x\right] \Delta x \\
& \quad+\frac{1}{2} \sum_{l=1}^{N}\left[\sum_{m=1}^{N} \eta_{m-1-l, l} u_{l}^{k} \lambda_{m}^{k} \Delta x\right] \Delta x \\
& \leq \frac{1}{2}\|\eta\|_{\infty}\left\|u^{k}\right\|_{\infty}\left\|u^{k}\right\|_{1}+\frac{1}{2}\left\|\eta_{x}\right\|_{\infty}\left\|u^{k}\right\|_{1}^{2}+\frac{1}{2}\|\eta\|_{\infty}\left\|\lambda^{k}\right\|_{1}\left\|u^{k}\right\|_{1}
\end{aligned}
$$

Further, using (2.7), (2.8), and (2.9) together with Lemma 2.1-Lemma 2.3 we get that there exists a positive constant $\Omega_{1}$ such that

$$
\frac{\left\|\lambda^{k+1}\right\|_{1}-\left\|\lambda^{k}\right\|_{1}}{\Delta t} \leq\left(\theta_{2}+2 B_{1}\right)\left\|\lambda^{k+1}\right\|_{1}+B_{1}\left\|\lambda^{k}\right\|_{1}+\Omega_{1} .
$$

This gives

$$
\left\|\lambda^{k+1}\right\|_{1}\left[1-\Delta t\left(\theta_{2}+2 B_{1}\right)\right] \leq\left\|\lambda^{k}\right\|_{1}\left(1+\Delta t B_{1}\right)+\Omega_{1} \Delta t
$$


which leads to

$$
\left\|\lambda^{k+1}\right\|_{1} \leq\left\|\lambda^{k}\right\|_{1}\left[\frac{1+\Delta t B_{1}}{1-\Delta t\left(\theta_{2}+2 B_{1}\right)}\right]+\frac{\Omega_{1} \Delta t}{\left[1-\Delta t\left(\theta_{2}+2 B_{1}\right)\right]} .
$$

Hence, there exists a constant $B_{2}$ such that $\left\|D_{h}^{-}\left(u^{k}\right)\right\|_{1}=\left\|\lambda^{k}\right\|_{1} \leq B_{2}$ for all $k=$ $1, \ldots, M$.

In the next result we will show that our approximations are Lipschitz continuous in time.

LEMmA 2.5. There exists an $L>0$, independent of $\Delta x$ and $\Delta t$ such that

$$
\sum_{j=1}^{N}\left|\frac{u_{j}^{m}-u_{j}^{p}}{\Delta t}\right| \Delta x \leq L(m-p) \text {. }
$$

Proof. From the first equation of the difference method (2.1) we get

$$
\begin{aligned}
& \sum_{j=1}^{N}\left|\frac{u_{j}^{k+1}-u_{j}^{k}}{\Delta t}\right| \Delta x \leq \sum_{j=1}^{N}\left|\frac{g_{j}^{k} u_{j}^{k+1}-g_{j-1}^{k} u_{j}^{k+1}+g_{j-1}^{k} u_{j}^{k+1}-g_{j-1}^{k} u_{j-1}^{k+1}}{\Delta x}\right| \Delta x \\
& +\sum_{j=1}^{N}\left|\frac{w_{j}}{Z} u_{j}^{k+1}\right| \Delta x+\frac{1}{2} \sum_{j=1}^{N}\left|\sum_{l=1}^{j} \eta_{j-l, l} u_{j-l}^{k} u_{l}^{k} \Delta x\right| \Delta x \\
& +\sum_{j=1}^{N}\left|u_{j}^{k+1} \sum_{l=1}^{N} \eta_{j, l} u_{l}^{k} \Delta x\right| \Delta x \\
& \leq \sum_{j=1}^{N}\left|\frac{g_{j}^{k}-g_{j-1}^{k}}{\Delta x}\right| u_{j}^{k+1} \Delta x+\sum_{j=1}^{N}\left|g_{j-1}^{k} \frac{u_{j}^{k+1}-u_{j-1}^{k+1}}{\Delta x}\right| \Delta x+\sum_{j=1}^{N}\left|\frac{w_{j}}{Z} u_{j}^{k+1}\right| \Delta x \\
& +\frac{1}{2} \sum_{j=1}^{N}\left|\sum_{l=1}^{j} \eta_{j-l, l} u_{j-l}^{k} u_{l}^{k} \Delta x\right| \Delta x+\sum_{j=1}^{N}\left|u_{j}^{k+1} \sum_{l=1}^{N} \eta_{j, l} u_{l}^{k} \Delta x\right| \Delta x \\
& \leq \theta_{2}\left\|u^{k+1}\right\|_{1}+\left\|\lambda^{k+1}\right\|_{1}\|g\|_{\infty}+\frac{1}{2} \sum_{j=1}^{N}\left|u_{j}^{k}\left(\sum_{l=1}^{N} \eta_{j, l} u_{l}^{k} \Delta x\right)\right| \Delta x \\
& +\sum_{j=1}^{N}\left|u_{j}^{k+1} \sum_{l=1}^{N} \eta_{j, l} u_{l}^{k} \Delta x\right| \Delta x \\
& \leq \theta_{2}\left\|u^{k+1}\right\|_{1}+\left\|\lambda^{k+1}\right\|_{1}\|g\|_{\infty}+B_{1}\left(2\left\|u^{k+1}\right\|_{1}+\left\|u^{k}\right\|_{1}\right) \text {. }
\end{aligned}
$$

From our earlier results, we arrive at

$$
\sum_{j=1}^{N}\left|\frac{u_{j}^{k+1}-u_{j}^{k}}{\Delta t}\right| \Delta x \leq L
$$

Hence, we obtain

$$
\sum_{j=1}^{N}\left|\frac{u_{j}^{m}-u_{j}^{p}}{\Delta t}\right| \Delta x \leq \sum_{k=p}^{m} \sum_{j=1}^{N}\left|\frac{u_{j}^{k+1}-u_{j}^{k}}{\Delta t}\right| \Delta x \leq L(m-p) .
$$

As in [14], we define a family of functions $\left\{U_{\Delta t, \Delta x}\right\}$ by $U_{\Delta t, \Delta x}(t, x)=u_{j}^{k}$ for $x \in$ $\left[x_{j-1}, x_{j}\right), t \in\left[t_{k-1}, t_{k}\right) j=1, \ldots, N$ and $k=1, \ldots, M$. Clearly, the set of functions 
$\left\{U_{\Delta t, \Delta x}\right\}$ is compact in the topology of $L^{1}\left((0, T) \times\left(0, x_{\max }\right)\right)$. Hence, the following result can be obtained.

LEMma 2.6. There exists a sequence $\left\{U_{\Delta t_{i}, \Delta x_{i}}\right\} \subset\left\{U_{\Delta t, \Delta x}\right\}$ which converges to a $B V\left([0, T] \times\left[0, x_{\text {max }}\right]\right)$ function $u(t, x)$ in the sense that $\forall t>0$ we have the following:

$$
\int_{0}^{x_{\max }}\left|U_{\Delta t_{i}, \Delta x_{i}}(t, x)-u(t, x)\right| d x \rightarrow 0
$$

and

$$
\int_{0}^{T} \int_{0}^{x_{\max }}\left|U_{\Delta t_{i}, \Delta x_{i}}(t, x)-u(t, x)\right| d x d t \rightarrow 0
$$

as $i \rightarrow \infty$. Furthermore, $u(t, x)$ satisfies $\|u\|_{B V\left([0, T] \times\left[0, x_{\max }\right]\right)} \leq B_{3}\left(\left|u^{0}\right|_{B V},|C|_{C^{1}}\right)$.

Proof. Same as in [14], p. 276, Theorem 16.7.

The next theorem will show that the limit function $u(t, x)$ constructed via our difference scheme is a weak solution of our partial differential equation (1.1).

Theorem 2.7. Any limit $u(t, x)$ defined in Lemma 2.6 is a weak solution of $(1.1)$ and satisfies

$$
\begin{aligned}
& \|u(t)\|_{1} \leq e^{\theta_{1} T}\left\|u^{0}\right\|_{1}+\int_{0}^{T} e^{\theta_{1}(T-s)}|C(s)| d s \\
& \|u\|_{L^{\infty}\left((0 . T) \times\left(0 . x_{\max }\right)\right)} \leq \max \left\{\left\|u^{0}\right\|_{\infty} e^{\left(\theta_{2}+\overline{B_{1}}\right) T}, \frac{\|C\|_{\infty}+\alpha_{2} \bar{P}}{\alpha_{1}}\right\}
\end{aligned}
$$

where $g(0, P) \geq \alpha_{1},|\beta(x, P)| \leq \alpha_{2}$ for $x \in\left[0, x_{\max }\right], P \in[0, \bar{P}]$ and

$$
\overline{B_{1}}=\max _{t \in[0 . T]} \frac{1}{2}\|\eta\|_{\infty}\|u(t)\|_{1} .
$$

Proof. We multiply Eq. (2.1) by $\phi_{j}^{k+1}=\phi\left(t_{k+1}, x_{j}\right)$ where $\phi \in C^{1}$. Then we multiply (2.1) by $\Delta x \Delta t$ and sum over our indices $j=1, \ldots, N$ and $k=1, \ldots, m$ and obtain

$$
\begin{aligned}
& \sum_{j=1}^{N}\left(u_{j}^{m+1} \phi_{j}^{m+1}-u_{j}^{0} \phi_{j}^{0}\right) \Delta x-\sum_{k=1}^{m} \phi_{0}^{k+1}\left(C^{k}+\sum_{j=1}^{N} \beta_{j}^{k} u_{j}^{k+1} \Delta x\right) \Delta t \\
& -\sum_{k=1}^{m} \sum_{j=1}^{N}\left(u_{j}^{k} \frac{\phi_{j}^{k+1}-\phi_{j}^{k}}{\Delta t}+g_{j-1}^{k} u_{j-1}^{k+1} \frac{\phi_{j}^{k+1}-\phi_{j-1}^{k+1}}{\Delta x}-\frac{w_{j}}{Z} u_{j}^{k+1} \phi_{j}^{k+1}\right) \Delta x \Delta t \\
& =\frac{1}{2} \sum_{k=1}^{m} \sum_{j=1}^{N} \phi_{j}^{k+1}\left(\sum_{l=1}^{j} \eta_{j-l . l} u_{j-l}^{k} u_{l}^{k} \Delta x\right) \Delta x \Delta t-\sum_{k=1}^{m} \sum_{j=1}^{N} \phi_{j}^{k+1} u_{j}^{k+1}\left(\sum_{l=1}^{N} \eta_{j . l} u_{l}^{k} \Delta x\right) \Delta x \Delta t .
\end{aligned}
$$

Using similar techniques to those used in the proof of Lemma 16.9, p. 279, in [14], one can easily show that the above equation converges to Eq. (1.2) along any convergent subsequence defined in Lemma 2.6. The bounds on the $L^{1}$ and $L^{\infty}$ norms of $u(t, \cdot)$ follow from those of $\left\{u^{k}\right\}$ in Lemma 2.2 and Lemma 2.3, respectively.

The following theorem shows the continuous dependence of the solution of (2.1) on the initial data. 
THEOREM 2.8. Let $\left\{u_{j}^{k}\right\}$ and $\left\{\widehat{u}_{j}^{k}\right\}$ be solutions of Eq. (2.1) corresponding to initial conditions $u_{j}^{0}$ and $\widehat{u}_{j}^{0}$ and boundary functions $C^{k}$ and $\widehat{C}^{k}$, respectively. Then, there exists a constant

$$
\begin{aligned}
& \gamma=\gamma\left(\max _{k}\left\|u^{k}\right\|_{1}, \max _{k}\left\|u^{k}\right\|_{\infty}, \max _{k}\left\|D_{h}^{-}\left(u^{k}\right)\right\|_{1}\right) \text { such that } \\
& {\left[1-\left(\theta_{1}+2 B_{1}\right) \Delta t\right]\left\|u^{k+1}-\widehat{u}^{k+1}\right\|_{1}} \\
& \quad \leq\left[1+\left(\gamma+4 B_{1}\right) \Delta t\right]\left\|u^{k}-\widehat{u}^{k}\right\|_{1}+\Delta t\left|C^{k}-\widehat{C}^{k}\right| \forall k \geq 0
\end{aligned}
$$

where $B_{1}$ is defined in Lemma 2.3 .

Proof. Following Lemma 16.6, p. 274 in [14], we set $v_{j}^{k}=u_{j}^{k}-\widehat{u}_{j}^{k}$ for $0 \leq k \leq M$ and $0 \leq j \leq N$. Then $v_{j}^{k}$ satisfies

$$
\begin{aligned}
& \frac{v_{j}^{k+1}-v_{j}^{k}}{\Delta t}+D_{h}^{-}\left(g\left(x_{j}, P^{k}\right) u_{j}^{k+1}-g\left(x_{j}, \widehat{P}^{k}\right) \widehat{u}_{j}^{k+1}\right)+\frac{w_{j}}{Z} v_{j}^{k+1} \\
& \quad=\frac{1}{2} \sum_{l=1}^{j} \eta_{j-l, l}\left(u_{j-l}^{k} u_{l}^{k}-\widehat{u}_{j-l}^{k} \widehat{u}_{l}^{k}\right) \Delta x-\sum_{l=1}^{N} \eta_{j, l}\left(u_{j}^{k+1} u_{l}^{k}-\widehat{u}_{j}^{k+1} \widehat{u}_{l}^{k}\right) \Delta x
\end{aligned}
$$

with the boundary condition

$$
\begin{aligned}
& g\left(0, P^{k}\right) u_{0}^{k+1}-g\left(0, \widehat{P}^{k}\right) \widehat{u}_{0}^{k+1}=\left(C^{k}-\widehat{C}^{k}\right)+\sum_{i=1}^{N} \beta_{i}^{k} v_{i}^{k+1} \Delta x \\
& +\sum_{i=1}^{N}\left(\beta\left(x_{i}, P^{k}\right)-\beta\left(x_{i}, \widehat{P}^{k}\right)\right) \widehat{u}_{i}^{k+1} \Delta x
\end{aligned}
$$

for $1 \leq j \leq N$. Multiplying each side of Eq. (2.10) by $\Delta x \operatorname{sgn}\left(v_{j}^{k+1}\right)$, summing over the indices $j=1, \ldots, N$ and using similar techniques as in the previous lemmas we obtain

$$
\left(1-\theta_{1} \Delta t\right)\left\|u^{k+1}-\widehat{u}^{k+1}\right\|_{1} \leq(1+\gamma \Delta t)\left\|u^{k}-\widehat{u}^{k}\right\|_{1}+\Delta t\left|C^{k}-\widehat{C}^{k}\right|+I+I I .
$$

The first term on the right-hand side of Eq. (2.10), after a transformation of variables, gives

$$
\begin{aligned}
I= & \frac{\Delta t}{2} \sum_{j=1}^{N} \sum_{l=1}^{N}\left(\eta_{j, l} u_{j}^{k} u_{l}^{k} \Delta x\right) \Delta x \operatorname{sgn}\left(v_{j}^{k+1}\right)-\frac{\Delta t}{2} \sum_{j=1}^{N} \sum_{l=1}^{N}\left(\eta_{j, l} \widehat{u}_{j}^{k} \widehat{u}_{l}^{k} \Delta x\right) \Delta x \operatorname{sgn}\left(v_{j}^{k+1}\right) \\
= & \frac{\Delta t}{2} \sum_{j=1}^{N} \sum_{l=1}^{N} \eta_{j, l} u_{j}^{k}\left(u_{l}^{k}-\widehat{u}_{l}^{k}\right) \Delta x\left(\Delta x \operatorname{sgn}\left(v_{j}^{k+1}\right)\right) \\
& \quad+\frac{\Delta t}{2} \sum_{j=1}^{N} \sum_{l=1}^{N} \eta_{j, l} \widehat{u}_{l}^{k}\left(u_{j}^{k}-\widehat{u}_{j}^{k}\right) \Delta x\left(\Delta x \operatorname{sgn}\left(v_{j}^{k+1}\right)\right) \\
\leq & \frac{1}{2}\|\eta\|_{\infty}\left\|u^{k}\right\|_{1}\left\|u^{k}-\widehat{u}^{k}\right\|_{1} \Delta t+\frac{1}{2}\|\eta\|_{\infty}\left\|\widehat{u}^{k}\right\|_{1}\left\|u^{k}-\widehat{u}^{k}\right\|_{1} \Delta t \\
= & \frac{1}{2}\|\eta\|_{\infty}\left(\left\|u^{k}\right\|_{1}+\left\|\widehat{u}^{k}\right\|_{1}\right)\left\|u^{k}-\widehat{u}^{k}\right\|_{1} \Delta t .
\end{aligned}
$$


Similarly, the second term on the right-hand side of (2.5) gives

$$
\begin{aligned}
& I I= \Delta t \sum_{j=1}^{N} \sum_{l=1}^{N} \eta_{j, l}\left(u_{j}^{k+1} u_{l}^{k}-\widehat{u}_{j}^{k+1} \widehat{u}_{l}^{k}\right) \Delta x\left(\Delta x \operatorname{sgn}\left(v_{j}^{k+1}\right)\right) \\
&=\Delta t \sum_{j=1}^{N} \sum_{l=1}^{N} \eta_{j, l} u_{j}^{k+1}\left(u_{l}^{k}-\widehat{u}_{l}^{k}\right) \Delta x\left(\Delta x \operatorname{sgn}\left(v_{j}^{k+1}\right)\right) \\
& \quad+\Delta t \sum_{j=1}^{N} \sum_{l=1}^{N} \eta_{j, l} \widehat{u}_{l}^{k}\left(u_{j}^{k+1}-\widehat{u}_{j}^{k+1}\right) \Delta x\left(\Delta x \operatorname{sgn}\left(v_{j}^{k+1}\right)\right) \\
& \leq\|\eta\|_{\infty}\left\|\widehat{u}^{k}\right\|_{1}\left\|u^{k+1}-\widehat{u}^{k+1}\right\|_{1} \Delta t+\|\eta\|_{\infty}\left\|u^{k+1}\right\|_{1}\left\|u^{k}-\widehat{u}^{k}\right\|_{1} \Delta t .
\end{aligned}
$$

Combining (2.11) and (2.12) we get

$$
\begin{aligned}
\left(1-\theta_{1} \Delta t\right)\left\|u^{k+1}-\widehat{u}^{k+1}\right\|_{1} & \leq(1+\gamma \Delta t)\left\|u^{k}-\widehat{u}^{k}\right\|_{1}+\Delta t\left|C^{k}-\widehat{C}^{k}\right| \\
& +\Delta t B_{1}\left(2\left\|u^{k+1}-\widehat{u}^{k+1}\right\|_{1}+4\left\|u^{k}-\widehat{u}^{k}\right\|_{1}\right) .
\end{aligned}
$$

This leads to

$$
\left[1-\Delta t\left(\theta_{1}+2 B_{1}\right)\right]\left\|u^{k+1}-\widehat{u}^{k+1}\right\|_{1} \leq\left[1+\Delta t\left(\gamma+4 B_{1}\right)\right]\left\|u^{k}-\widehat{u}^{k}\right\|_{1}+\Delta t\left|C^{k}-\widehat{C}^{k}\right|,
$$

which is the required result.

3. Uniqueness of the BV weak solution. To complete our task, we need to show that the weak solution to our model, given in (1.2) is unique. From this we can conclude that the finite difference scheme converges to the unique solution of our equation. To obtain such a result, we first consider the nonautonomous semilinear problem (1.1), where we treat $P(t) \in C^{1}(0, T)$ as a given function (i.e., we do not impose the compatibility condition $\left.P(t)=\int_{0}^{x_{\max }} u(t, x) d x\right)$. Then it is easily established that Eq. (1.1) has a unique weak solution in $C\left(0, T ; L^{1}\left(0, x_{\max }\right)\right)$. Note that the existence of such a weak solution follows immediately from our previous analysis. In fact, such a solution can be defined to be the limit of our finite difference scheme, by treating $P^{k}=P\left(t_{k}\right)$ as a given number. Hence, we need only to show that this weak solution is unique. To establish this result, we consider techniques used in [14] which are based on a nonlinear version of the Holmgren uniqueness theorem. To this end, assume that $u$ and $v$ are two weak solutions of such an equation and define $h=u-v$. Multiplying by a test function $\phi$ and 
integrating, one can easily obtain the following relation using integration by parts:

$$
\begin{aligned}
\int_{0}^{x_{\max }} & h(T, x) \phi(T, x) d x \\
= & \int_{0}^{T} \int_{0}^{x_{\max }} h\left(\phi_{t}+g \phi_{x}-\frac{w}{Z} \phi\right) d x d t \\
& +\int_{0}^{T} \phi(t, 0) \int_{0}^{x_{\max }} \beta(x, P(t)) h(t, x) d x d t \\
& +\int_{0}^{T} \int_{0}^{x_{\max }}(F(u)-F(v)) \phi d x d t .
\end{aligned}
$$

We simplify the last term by using techniques such as changing the order of integration, together with (H8), to obtain

$$
\begin{aligned}
& \int_{0}^{T} \int_{0}^{x_{\max }}(F(u)-F(v)) \phi d x d t \\
& \quad=\int_{0}^{T} \int_{0}^{x_{\max }} h(t, x)\left(\frac{1}{2} \int_{0}^{x_{\max }-x} \phi(t, x+y) \eta(x, y)(u(t, y)+v(t, y)) d y\right. \\
& \left.\quad-\int_{0}^{x_{\max }} \phi(t, y) \eta(x, y) v(t, y) d y-\phi(t, x) \int_{0}^{x_{\max }} \eta(x, y) u(t, y) d y\right) d x d t
\end{aligned}
$$

We now set up the following backward problem:

$$
\left\{\begin{array}{l}
\phi_{t}+g(x, P(t)) \phi_{x}-\frac{w(x)}{Z} \phi=\psi(t, x)+F^{*}(\phi), \\
\phi(t, 0)=0, \quad t \in(0, T), \\
\phi(T, x)=a(x), \quad x \in\left[0, x_{\max }\right]
\end{array}\right.
$$

where

$$
\begin{aligned}
F^{*}(\phi)=\frac{1}{2} \int_{0}^{x_{\max }-x} \phi(t, x+y) \eta(x, y)(u(t, y)+v(t, y)) d y \\
\quad-\int_{0}^{x_{\max }} \phi(t, y) \eta(x, y) v(t, y) d y \\
-\phi(t, x) \int_{0}^{x_{\max }} \eta(x, y) u(t, y) d y
\end{aligned}
$$


Since $F^{*}$ is a linear mapping in $\phi$ it can easily be shown that the linear PDE (3.2) has a unique solution $\phi \in C^{1}\left([0, T] \times\left[0, x_{\max }\right]\right)$ for any function $a$ lying in some dense subspace of $L^{2}\left(0, x_{\max }\right)$. Hence, this establishes the uniqueness of a solution to Eq. (1.1) given the function $P(t)$. This would imply that our finite difference solution to (2.1) given $P^{k}=P\left(t_{k}\right)$ converges to the unique solution of Eq. (1.1) where $P(t) \in C^{1}(0, T)$. Also, from the proof of Theorem 2.8 where $\left\{u^{k}\right\}$ and $\left\{\widehat{u}^{k}\right\}$ are the solutions of Eq. (2.1) corresponding to $\left(C^{k}, P^{k}\right)$ and $\left(\widehat{C}^{k}, \widehat{P}^{k}\right)$ respectively, we have

$$
\left\|v^{k+1}\right\|_{1} \leq\left(\frac{1}{\left[1-\left(\theta_{1}+2 B_{1}\right) \Delta t\right]}\right)\left\|v^{k}\right\|_{1}+\frac{\left(\gamma+4 B_{1}\right) \Delta t\left|P^{k}-\widehat{P}^{k}\right|+\Delta t\left|C^{k}-\widehat{C}^{k}\right|}{\left[1-\left(\theta_{1}+2 B_{1}\right) \Delta t\right]}
$$

where $v^{k}=u^{k}-\widehat{u}^{k}$. This leads to

$$
\begin{aligned}
\left\|v^{k}\right\|_{1} & \leq\left\|v^{0}\right\|_{1}\left(\frac{1}{\left[1-\left(\theta_{1}+2 B_{1}\right) \Delta t\right]}\right)^{k} \\
& +\sum_{j=1}^{k}\left[1-\left(\theta_{1}+2 B_{1}\right) \Delta t\right]^{k-j}\left[\left(\gamma+4 B_{1}\right)\left|P^{j}-\widehat{P}^{j}\right|+\left|C^{j}-\widehat{C}^{j}\right|\right] \Delta t,
\end{aligned}
$$

where $\Delta t$ can be chosen so that $\left(\theta_{1}+2 B_{1}\right) \Delta t<1$. From Theorem 2.7, $\left\{U_{\Delta t, \Delta x}\right\}$ converges to $u(t, x)$ and $\left\{\widehat{U}_{\Delta t, \Delta x}\right\}$ to $\widehat{u}(t, x)$ strongly in $C\left(0, T ; L^{1}\left(0, x_{\max }\right)\right)$. Taking the limit in Eq. (3.3) we arrive at the following estimate:

$$
\begin{aligned}
\|v(t)\|_{1} \leq & e^{\left(\theta_{1}+2 B_{1}\right) t}\|v(0)\|_{1} \\
& \quad+\int_{0}^{t} e^{\left.\left(\theta_{1}+2 B_{1}\right), t-s\right)}\left[\left(\gamma+4 B_{1}\right)|P(s)-\widehat{P}(s)|+|C(s)-\widehat{C}(s)|\right] d s
\end{aligned}
$$

where $v(t, x)=u(t, x)-\widehat{u}(t, x)$ and $u(t, x)$ and $\widehat{u}(t, x)$ are the unique solutions of (1.1) given $(C(t), P(t))$ and $(\widehat{C}(t), \widehat{P}(t))$, respectively. Applying the above estimate to $P(t)=$ $\int_{0}^{x_{\max }} u(t, x) d x$ and $\widehat{P}(t)=\int_{0}^{x_{\max }} \widehat{u}(t, x) d x$ we get the following theorem.

THEOREM 3.1. Any two solutions $u$ and $\widehat{u}$ of Eq. (1.1) satisfy the following:

$$
\|u(t)-\widehat{u}(t)\|_{1} \leq e^{\left(\theta_{1}+\gamma+6 B_{1}\right) t}\|u(0)-\widehat{u}(0)\|_{1}+\int_{0}^{t} e^{\left(\theta_{1}+\gamma+6 B_{1}\right)(t-s)}|C(s)-\widehat{C}(s)| d s
$$

where $\gamma$ and $B_{1}$ are as defined earlier in this paper. Hence, the Bounded Variation weak solution $u(t, x)$ to Eq. (1.1) is unique because $C$ and $u^{0}$ are fixed functions in the initial-boundary value problem (1.1).

4. Numerical results. We check the accuracy of our algorithm using an example illustrated in this section. Let the parameters $g, w, \beta, \eta$ be given as follows:

$$
\begin{aligned}
& g(x, P)=(1-x) 0.5 e^{-0.2 P}, \quad \beta(x, P)=0.5 x e^{-0.2 P}, \quad w(x)=0.5 \\
& \eta(x, y)=1-(x+y), \quad C(t)=0 \quad \text { and } Z=1 .
\end{aligned}
$$


Then, our model reduces to the following form:

$$
\begin{aligned}
u_{t}+0.5 e^{-0.2 P}[(1-x) u]_{x}+0.5 u & =\frac{1}{2} \int_{0}^{x}(1-x) u(t, x-y) u(t, y) d y \\
& -\int_{0}^{1}[1-(x+y)] u(t, x) u(t, y) d y, \\
u(t, 0) & =\int_{0}^{1} x u(t, x) d x, \\
u(0, x) & =u^{0}(x) .
\end{aligned}
$$

We integrate Eq. (4.1) with respect to $x$ and use integration by parts to get the following ordinary differential equation:

$$
\frac{d P}{d t}=0.5 e^{-0.2 P} Q-\frac{P^{2}}{2}+P Q-0.5 P
$$

where we define

$$
Q(t)=\int_{0}^{1} x u(t, x) d x
$$

Multiplying (4.1) by $x$ and integrating with respect to $x$ again and using integration by parts here as well, we obtain the second of our system of two coupled equations, namely

$$
\frac{d Q}{d t}=0.5 e^{-0.2 P}(P-Q)-0.5 Q
$$

Here we have used the fact that $\int_{0}^{1} x F(u) d x=0$. We choose the initial condition

$$
u^{0}(x)= \begin{cases}1 & x \in[0,0.1] \\ 0 & x \in(0.1,1]\end{cases}
$$

We assume that the exact solution is the numerical solution to the ordinary differential equation which we obtain using a $4-5^{\text {th }}$ order Runge-Kutta routine available in MATLAB. We compare the total population $P^{\Delta x}(t)$ and the total biomass $Q^{\Delta x}(t)$ using the algorithm described in Sec. 2, with $\Delta x=0.01$ and $\Delta t=0.01$, to the total population $P(t)$ and total biomass $Q(t)$ that result from the differential equation solution, and present the results in Figures 1 and 2, respectively.

In Figure 3 we present the following relative errors:

$$
\begin{aligned}
& \sup _{t \in[0,1]} \frac{\left|P^{\Delta x}(t)-P(t)\right|}{|P(t)|}, \\
& \sup _{t \in[0,1]} \frac{\left|Q^{\Delta x}(t)-Q(t)\right|}{|Q(t)|}
\end{aligned}
$$

as functions of $\Delta x$. The results in Figure 3 indicate a first-order convergence, as expected. 


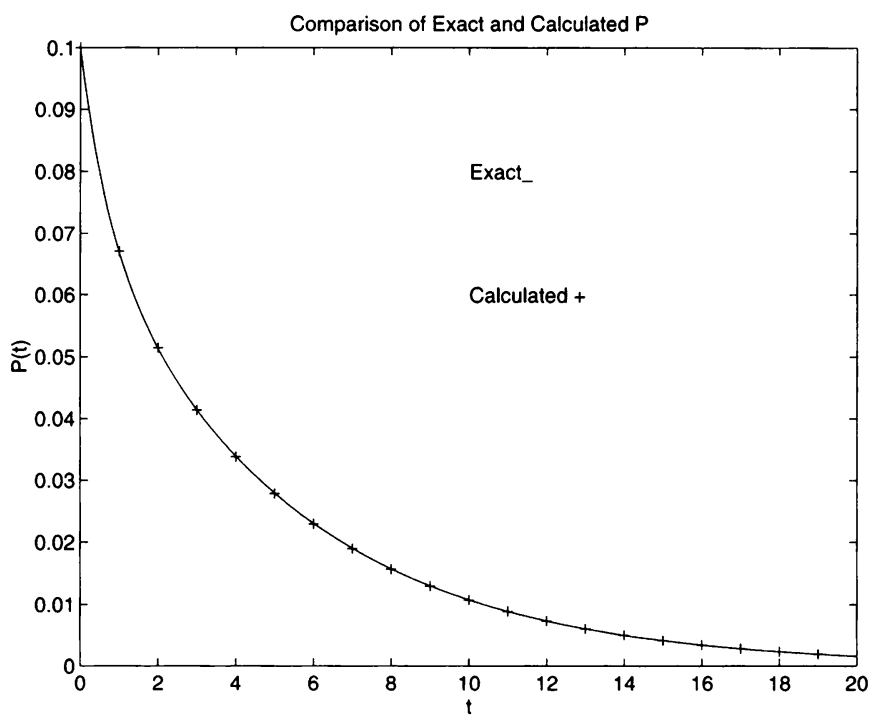

Fig. 1. A comparison between Exact and Calculated P.

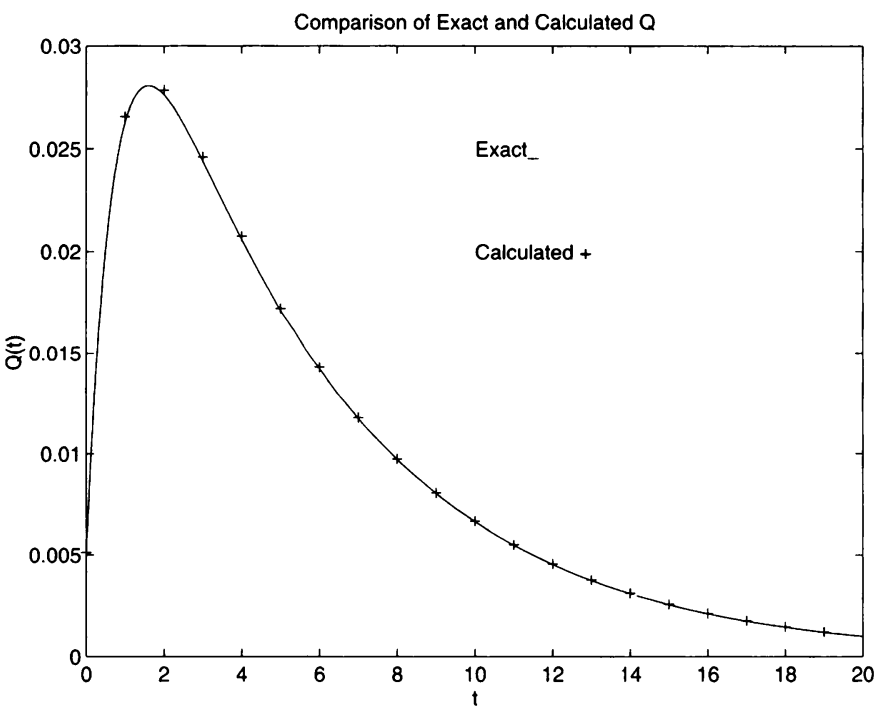

FIG. 2. A comparison between Exact and Calculated Q. 


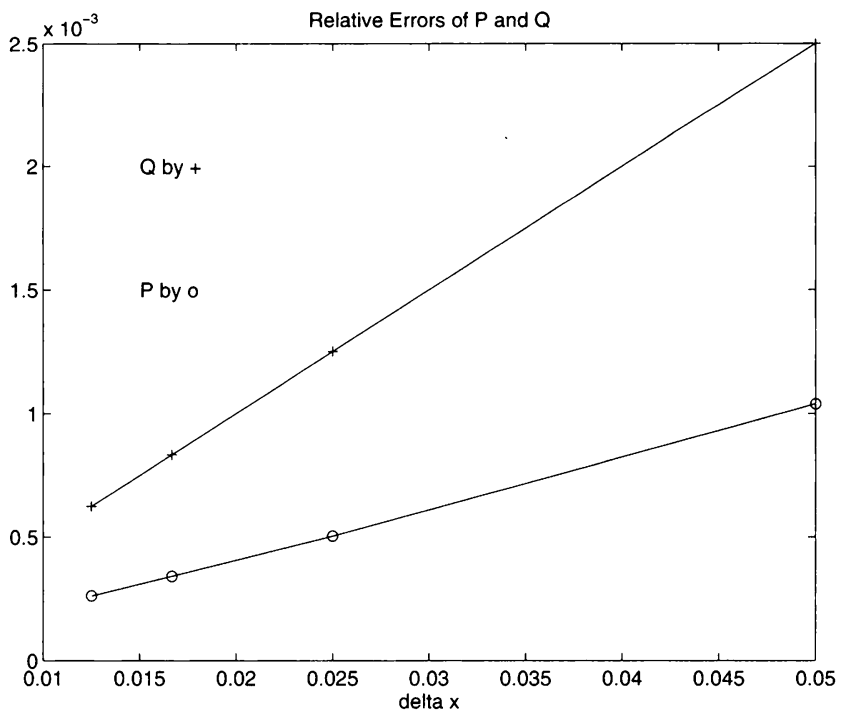

FIG. 3. A representation of the relative errors of $P$ and $Q$, with respect to $\Delta x$.

Acknowledgments. This work is supported by LEQSF grant \# LEQSF (1996-99)RD-A-36.

\section{REFERENCES}

[1] A. S. Ackleh, Parameter Estimation in a Structured Algal Coagulation Fragmentation Model, Nonlinear Analysis, Theory Methods and Applications 28, 837-854 (1997)

[2] A. S. Ackleh and B. G. Fitzpatrick, Modeling Aggregation and Growth Processes in an Algal Population Model: Analysis and Computation, Journal of Mathematical Biology 35, 480-502 (1997)

[3] A. S. Ackleh, B. G. Fitzpatrick, and T. G. Hallam, Approximation and Parameter Estimation Problems for Algal Aggregation Models, Mathematical Models and Methods in Applied Sciences 4, 291-311 (1994)

[4] A. S. Ackleh, T. G. Hallam, and Helene C. Muller Landau, Estimation of Sticking and Contact Efficiencies in Aggregation of Phytoplankton: The 1993 SIGMA Tank Experiment, Deep-Sea Research II, 42, 185-201 (1995)

[5] A. S. Ackleh, T. G. Hallam, and W. O. Smith, Influences of Aggregation and Grazing on Phytoplankton Dynamics and Fluxes: An Individual Based Modeling Approach, Nonlinear World 1, 473-492 (1994)

[6] A. S. Ackleh and K. Ito, An Implicit Finite Difference Scheme for the Nonlinear Sized-Structured Population Model, Numerical Functional Analysis and Optimization 18, 865-884 (1997)

[7] M. G. Crandall and Andrew Majda, Monotone Difference Approximations for Scalar Conservation Laws, J. Math. Comp. 34, 1-21 (1980)

[8] G. A. Jackson, Compared Observed Changes in Particle Size Spectra with Those Predicted Using Coagulation Theory, Deep-Sea Research II, 42, 159-184 (1994)

[9] G. Jackson, A Model of Formation of Marine Algal Flocs by Physical Coagulation Processes, DeepSea Research 37, 1197-1211 (1990)

[10] I. N. McCave, Size-Spectra and Aggregation of Suspended Particles in the Deep Ocean, Deep-Sea Research 31, 329-352 (1984)

[11] C. R. O'Melia and K. S. Bowman, Origins and Effects of Coagulation in Lakes, Schweiz. Z. Hydrol. 46, 64-85 (1984)

[12] T. R. Parsons and M. Takahashi, Biological Oceanographic Processes, Pergamon Press, 1984 
[13] U. Riebesell and D. A. Wolf-Gladrow, The Relationship between Physical Aggregation of Phytoplankton and Particle Flux: A Numerical Model, Deep-Sea Research 39, 1085-1102 (1992)

[14] J. Smoller, Shock Waves and Reaction-Diffusion Equations, Springer-Verlag, New York, 1994

[15] U. Weilenmann, C. R. O'Melia, and W. Stumm, Particle Transport in Lakes: Models and Measurements, Limnology and Oceanography 34, 1-18 (1989)

[16] D. M. Young, Iterative Solutions of Large Linear Systems, Academic Press, New York and London, 1971 